\title{
Fabrication of Poly (sebacic acid-co-ricinoleic-ester anhydride) with $\beta$-cyclodextrin-loaded doxorubicin Implants and in vitro characterization
}

\author{
Nagaraja Sreeharsha ${ }^{1,9, *}$, Jagadeesh G Hiremath², Bandar E AI-Dhubiab', Girish Meravanige ${ }^{3}$, Ranjith \\ Kumar Karnati ${ }^{4}$, Mahesh Attimarad ${ }^{1}$, Anroop B Nair ${ }^{1}$, Katharigatta N Venugopal ${ }^{1,10}$, Mohamed A \\ Morsy $^{1,8}$, Shinu Pottathil ${ }^{5}$, Saleemmulla Khan ${ }^{6}$, Nagashekhara Molugulu ${ }^{7}$
}

\begin{abstract}
1'Department of Pharmaceutical Sciences, College of Clinical Pharmacy, King Faisal University, Al-Ahsa, SAUDI ARABIA ${ }^{2}$ Department of Pharmaceutics, PA College of Pharmacy, Mangalore, Karnataka, INDIA.

${ }^{3}$ Department of Biomedical Sciences, College of Medicine, King Faisal University, Al-Ahsa, SAUDI ARABIA.

${ }^{4}$ Department of Chemistry, College of Science, King Faisal University, Al-Ahsa, SAUDI ARABIA.

${ }^{5}$ Department of Biomedical Sciences, College of Clinical Pharmacy, King Faisal University, Al-Ahsa, SAUDI ARABIA.

${ }^{6}$ Department of Pharmacognosy, PA College of Pharmacy, Mangalore, Karnataka, INDIA.

${ }^{7}$ School of Pharmacy, Monash University, Jalan Lagoon Selatan, Bandar Sunway, Selangor, MALAYSIA.

${ }^{8}$ Department of Pharmacology, Faculty of Medicine, Minia University, El-Minia 61511, EGYPT.

${ }^{9}$ Department of Pharmaceutics, Vidya Siri College of Pharmacy, Off Sarjapura Road, Bangalore, INDIA.

${ }^{10}$ Department of Biotechnology and Food Technology, Durban University of Technology, Durban, SOUTH AFRICA.
\end{abstract}

\begin{abstract}
Background: Doxorubicin is an excellent molecule for the formation of biodegradable implants using the poly (sebacic acid-co-ricinoleic-ester anhydride) 70:30 w/w (poly[SARA] 70:30 w/w PSRA 7/3) polymer. Methods: The cylindrical implants were successfully produced by means of hot melt extrusion. We used differential scanning calorimetric (DSC) and X-ray diffraction (XRD) methods to identify the melting state and crystal type of blank and drug-loaded implants. A study was conducted on PSRA $7 / 3 \mathrm{w} / \mathrm{w}$ blank and drug-loaded implants for in vitro hydrolytic degradation. The drug present in the remaining sample was estimated. Results: The hydrolytic degradation rate of the $10 \%$ $\mathrm{w} / \mathrm{w}$ (F2)-loaded implant was relatively low when compared with the $20 \% \mathrm{w} / \mathrm{w}$ (F3) implant. Discussion: In vitro drug release studies illustrated that the drug-release rate was faster in association with increasing amounts of doxorubicin in the implant.
\end{abstract}

Key words: Poly(sebacic acid-co-ricinoleic-ester anhydride) 70:30 w/w, Doxorubicin, $\beta$-cyclodextrin, Cylinder, Biodegradable polymer, in vitro.

\section{INTRODUCTION}

Biodegradable polymers are used in the production of implantable devices and drug carriers and as scaffolds in regenerative medicine. They are different from biodegradable polymer biomaterials since they degrade via hydrolysis or enzymatic processes and are eliminated or absorbed. They do not need to be replaced, nor do they require surgical intervention. Polyanhydrides have been utilized as carriers in the controlled delivery of drugs. Today, polyanhydride polymers are the "materials of advanced drug delivery systems. ${ }^{1-3}$
In 1983, Langer recognized a polyanhydride class of hydrolytically unstable polymers that was suitable for controlled drugrelease. ${ }^{4-5}$ Later, Domb et al. synthesizedsebacic acid $(\mathrm{SA})$, ricinoleic acid (RA) and poly ([carboxy phenoxy] propane-sebacic acid)(PCPP-SA). ${ }^{6}$ The established pharmacological effects of polyanhydride polymer-formed drug carriers include their biocompatibility and safety, as well as their non-toxic, nonmutagenic and low-teratogenic properties. ${ }^{7}$ Marketed products composed of hydroxy stearic acid (HPA)-based polyanhydride
Submission Date: 29-02-2020; Revision Date: 30-03-2020; Accepted Date: 21-05-2020

DOI: 10.5530/ijper.54.3.135 Correspondence: Dr. Nagaraja SreeHarsha Department of

Pharmaceutical Sciences, College of Clinical Pharmacy, King Faisal University, Al-Ahsa-31982, SAUDI ARABIA.

Phone: +96 6535485322

E-mail: sharsha@kfu.edu.sa

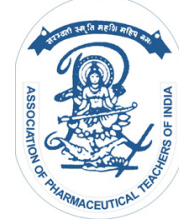

www.ijper.org 
drug carrier systems are available for the treatment of brain cancer and osteomyelitis and includeagents such

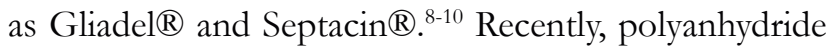
polymers gained much interest in the preparation and development of controlled drug-delivery systems. These drug-delivery systems include implants ${ }^{11,12}$ pastes (injectables), ${ }^{13}$ microspheres ${ }^{14}$ and nanomedicine ${ }^{15,5}$ and target-specific drug-delivery systems. ${ }^{16}$ It has been well described that without the need for any pharmaceutical excipients, fatty acid polymers of polyanhydride polymers undergo surface erosion due the effects associated with unstable anhydride bonds and the presence of an unstable backbone, such as SA and RA. However, erosion is a rate-limiting step to ensuring drug release from the polymeric matrix. ${ }^{17,13}$ The drug released from any carrier system is not dependent on its physicochemical properties and drug release can be controlled by alterations in SA or RA in different proportions, or it can be affected by modifications to the microstructure. The polyanhydrides are so important in developing drug-delivery systems. Specifically, it is predicted that polyanhydride-based drug carriers undergo surface erosion given the significant water labiality of anhydride bonds inhibited water penetration thus results in bulking and hydrophobicity. ${ }^{18} \mathrm{~A}$ reduction in the material thickness throughout the surface disintegration process of cylindrical shape approximately zero-order hydrolytic degradation kinetics illustrate the heterogeneous surface erosion. ${ }^{18}$ One characteristic property of the high hydrolytic reactivity of anhydride linkages is its adaptability to manage degradation rates. This type of control can be manipulated by varying the type and proportions of the monomer used. The ability to synthesize particular kinds of polymers can result in the predictable release of a drug from a formulation in over the course of 1 week to several weeks. By changing the hydrophobichydrophilic balance of the polymer, basic changes in the polymer backbone occur and hydrolytic degradation can be controlled. A delay in degradation amounts of aliphatic and aromatic polyanhydride polymers were few days to months. Thus, polyanhydride provides a convenient method through which to produce a drug discharge occur by a sequence of the erosion of the matrix, diffusion through the polymer matrix and/or diffusion through pores formed in the matrix by the dissolution of the water-soluble additives. ${ }^{2,19,20}$

PSRA7/3 w/w is a polyanhydride class of polymers used in controlled drug-carrier systems. Doxorubicin is an ideal molecule for the preparation of biodegradable implants using the PSRA7/3 w/w polymer. Doxorubicin is one of the most potent, broad-spectrum anticancer agents to be used as a therapeutic option for solid tumors. Drugs have a short half-life due to this narrow therapeutic index and they also demonstrate significant increases in high-dose distributions to healthy normal tissues. Drug distribution leads to the onset of drug-related side effects, such as cardiotoxicity, myelosuppression and mucositis. ${ }^{21,22}$ Hence, localized drug delivery systems are needed for doxorubicin, which has many applications and offers promising results as a potential solution to the significant drawbacks associated with some other currently available drug-delivery systems. Polyanhydride-based, localized drug-release systems (such as insoluble drugs in aqueous media) have low bioavailability and a short half-life, meaning that the drug can be delivered directly to the desired site. These delivery techniques are useful for drugs with a limited therapeutic index. Local drug delivery is suitable for medications that exert dosage-reliant action; this prevents the need to use other drugs that have adverse effects when delivered systemically. Local delivery further reduces dose-related problems that are typically encountered during animal studies and clinical studies with humans. With respect to anticancer drugs, intratumoral delivery is unrestricted by insufficient blood supply, which is induced by radiation therapy or surgery. Polyanhydrides also demonstrate reduced liability before delivering the active agent due its surface or heterogeneous hydrolytic degradation. ${ }^{23}$ Hence, the present study explores the preparation of DOXHClloaded biodegradable implants using a PSRA7/3 w/w polymer and also features in vitro characterization studies.

\section{MATERIALS AND METHODS}

Doxorubicin (DOXHCl) was obtained as a free product from RPG Life Sciences Ltd. (Mumbai, India). Poly (sebacic acid-co-ricinoleic-ester anhydride) 70:30 w/w (poly[SA-RA]70:30 w/w, designated as PSAR 7/3 $\mathrm{w} / \mathrm{w}$ ) (monomer number $[\mathrm{Mn}]=21,000$, molecular weight $[\mathrm{Mw}]$ of 60,000 and a melting temperature $\left[\mathrm{mT}^{\circ} \mathrm{C}\right]=68.8$, data $\left.\mathrm{HJ} / \mathrm{gr}\right]^{\circ} \mathrm{C}$ ), was procured from the School of Pharmacy, Faculty of Medicine, the Hebrew University of Jerusalem, Hadassah Ein Kerem Medical Center Campus, Jerusalem 91120, Israel. It was used without further purification. Complete information on the polymers used is available. ${ }^{13}$ Beta $\beta$-cyclodextrin $(\mathrm{mW} \mathrm{1,134.98)}$ and all analytical grade solvents were procured from Sigma Aldrich Co. (St. Louis, MO, USA).

\section{Preparation of DOX.HCI Implants}

$\mathrm{DOX} \cdot \mathrm{HCl}$-loaded cylindrical polyanhydride-based implants were prepared using a method published previously. ${ }^{24}$ In short, we employed a laboratory-based 
method to produce the implants. For the first step, the drug and $\beta$-cyclodextrin were mixed; a pasty polymer was uniformly blended and brought into a cylindrical mold (7.0 $\mathrm{mm}$ in diameter and $8.0 \mathrm{~mm}$ in length). It was set on and attached to a cylinder (rod) of $6.9 \mathrm{~mm}$ in diameter (portable). The full component was heated to $50^{\circ} \mathrm{C}$ using a heating mantle. The polymer was plasticized and homogenously mixed using a steel rod (length $10 \mathrm{~mm} \times$ breath $2 \mathrm{~mm}$ ). After homogenous mixing, the full unit was placed in a freezer $\left(-4^{\circ} \mathrm{C}\right)$. After $5 \mathrm{~min}$, the unit was removed and a plunger was introduced into the mold; a cylindrical shape was created and manually compressed using a flexible plunger, which was pushed $1.0-7.0 \mathrm{~mm}$ into the mold. The cylinder was returned to the freezer for $20 \mathrm{~min}$. After $20 \mathrm{~min}$, the whole unit was left to return to room temperature. At this point, the plunger and rod were drawn out, which extruded the solidified implant from the mold.

\section{Physicochemical analysis of the implants}

The area $\left(\mathrm{cm}^{2}\right)$, thickness $(\mathrm{h}, \mathrm{cm})$, volume $\left(\mathrm{cm}^{2}\right)$ and surface area $\left(\mathrm{cm}^{2}\right)$ of the implants were measured using a digital vernier caliper. From the obtained values, the above dimensions were measured using the mathematical equations $^{17}$ shown in Table 1 . We used differential scanning calorimetry (DSC) and X-ray diffraction (XRD) methods to detect the melting state and crystal structure of the blank and drug-loaded implants. DSC employing the Mettler Toledo DSC822e (Columbus, $\mathrm{OH}$, USA) was used to investigate the thermal properties of the samples at a rate of $10^{\circ} \mathrm{C}$ per min, with temperatures ranging from $10^{\circ} \mathrm{C}$ to $230^{\circ} \mathrm{C}$. It used a An empty aluminum skillet was used as a reference. XRD examinations of the implants were carried out using a Philips PW1820 (Amsterdam, the Netherlands) with $\mathrm{Cu} \mathrm{Ka} \alpha$ and $\mathrm{Ka} \alpha$ radiation to identify the crystal patterns. The lyophilized samples were sputtered coated with gold and the morphology of the implants was visualized by scanning electron microscope (SEM; JSM-848, JEOL, Tokyo, Japan). The generated surface morphology of the coated cross-section samples was examined under magnification.

\section{$\mathrm{DOX} \cdot \mathrm{HCl}$ content analysis in the implants}

Implants loaded with $10 \mathrm{mg}$ of $\mathrm{DOX} \cdot \mathrm{HCl}$ were dissolved in $5 \mathrm{~mL}$ of chloroform (CHL) combined with $10 \mathrm{~mL}$ of MillliQ water until it turned an opaque white color to ensure precipitation of the polymer. The samples were centrifuged at $4^{\circ} \mathrm{C}$ (Remi, Bengaluru, India) at 12,000 rpm for $12 \mathrm{~min}$. The collected supernatant was clear and subsequently evaporated. After evaporating the CHL, 5 $\mathrm{mL}$ of phosphate buffer saline (PBS; $\mathrm{pH}$ 7.4) was added and vortex stirring was performed for $5 \mathrm{~min}$. A $0.22 \mu \mathrm{m}$ filter was used (Millipore India). The DOX $\cdot \mathrm{HCl}$ content was analyzed with an ultraviolet visible (UV-VIS)spectrophotometer (UV-1800; Shimadzu, Kyoto, Japan) at $250 \mathrm{~nm}$. This analysis procedure was standardized and validated. The correlation coefficient $(r 2)$ is 0.9998 and the equation was $y=0.012 x+0.004$ with a slope of 0.012 and an intercept of 0.004 . This method to assess $\mathrm{DOX} \cdot \mathrm{HCl}$ has been developed and was found to be highly precise and valid. This method was successfully adopted in alternative evaluations.

\section{Hydrolytic degradation of implants}

An investigation was carried out on PSARA $7 / 3 \mathrm{w} / \mathrm{w}$ blank and drug-loaded implants to examine in vitro hydrolytic degradation and to investigate the amount of drug present in the remaining sample. The implants were placed in $20 \mathrm{~mL}$ of PBS (pH 7.4) in $50 \mathrm{mLscrew}$-capped vials. These vials were maintained in a horizontal shaker and vibrated at a velocity of $50 \mathrm{rpm}$ to activate the hydrolytic degradation for 20 days. At precise intervals, the blank and drug-loaded implants were collected from the media. These samples were lyophilized the implant weight was subsequently determined. For each sample, the vehicle in the flask was replaced with new buffer to promote the sink condition. The hydrolysis of the implant was measured by means of weight reduction and at each stage, the $\mathrm{DOX} \cdot \mathrm{HCl}$ content assembled in the implant (residue) was analyzed using the UV-VIS spectrophotometric method described above.

\section{In vitro PSRA7/3 w/w release studies of DOX $\cdot \mathrm{HCl}-$ loaded implants}

In vitro PSRA $7 / 3 \mathrm{w} / \mathrm{w}$ release studies of the hydrolytic, degradable drug carrying the implants were carried out using PBS ( $\mathrm{pH} 7.4$ ) as a dissolution medium. The implants were placed in $20 \mathrm{~mL}$ of PBS (pH 7.4) in $50 \mathrm{~mL}$ screw-capped vials. These vials were kept in a horizontal shaker and the samples were vibrated at a velocity of $50 \mathrm{rpm}$ to promote drug release for40 days. At each sampling time, the dissolution media were changed in the flask with fresh buffer to continue the sink condition. The $\mathrm{DOX} \cdot \mathrm{HCl}$ content was determined using the UV-VIS spectrophotometric method described above. The cumulative amount released versus time was plotted. Finally, we determined the amount of drug present based on kinetic models.

\section{RESULTS AND DISCUSSION}

\section{Implant Characterization}

Figure 1 provides photographs of the drug-loaded implants prepared using the melting method. The 
implants that were produced were of cylindrical shape with a smooth surface. Table 1 that the weight of the implants was as follows: $120.46 \pm 1.98 \mathrm{mg}$ (F1),

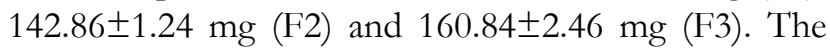
implant thickness was as follows: $2.06 \pm 0.01 \mathrm{~mm}$, $2.24 \pm 0.02 \mathrm{~mm}$ and $3.06 \pm 0.01 \mathrm{~mm}$. The drug content uniformity was found to be $98 \%-99 \%$. Figure 2 depicts the DSC studies that indicated a sharp endothermic peak of the drug measured at $209.83^{\circ} \mathrm{C}$; thePSRA $7 / 3$ $\mathrm{w} / \mathrm{w}$ endothermic peak was generated at $62.83^{\circ} \mathrm{C}$ and the $\beta$-cyclodextrin short, wide melting peak occurred at $58.86^{\circ} \mathrm{C}$. Figure 3 shows that based on the XRD analysis, the $2 \theta$ scattered angle peaks of the crystalline drug occurred at 17.27 and 18.46 and that their conforming peak intensities were 1,346 and 1,408, respectively. XRD analysis of the blank implant showed that the $2 \theta$ scattered angle peaks occurred at 21.73 and 21.91, with peak intensities at 248 and 196, respectively, while the $\beta$-cyclodextrin $2 \theta$ scattered angle peak appeared at 5.38 with an intensity peak at 4,568 .

\section{Hydrolytic polymer weight loss and drug accumulation}

Figure 4 shows the investigated hydrolytic degradation and drug accumulation in the remaining drug sample carried by the PSAR 7/3 w/w implants. The blank implants exhibited a faster hydrolytic degradation rate of basic mass loss $(4.78 \%)$ and at the end of 20 days, $96.63 \% \pm 1.9 \%$ degraded. The hydrolytic degradation rate of the $10 \% \mathrm{w} / \mathrm{w}$ (F2)-loaded implant was relatively lower when linked to the $20 \% \mathrm{w} / \mathrm{w}$ (F3) implant. After 2 days, degradation mass loss took place $(2.64 \% \pm 0.9 \%$ and $6.64 \% \pm 1.2 \%)$. During the course of this degradation, we found that the quantity of the drug was $99.45 \pm 0.8$ and $98.46 \pm 0.4$. At the end of a 20 -day study, the resulting mass loss of the drug implant (F2) produced a quantity of $76.96 \pm 1.2$; however, at this stage,

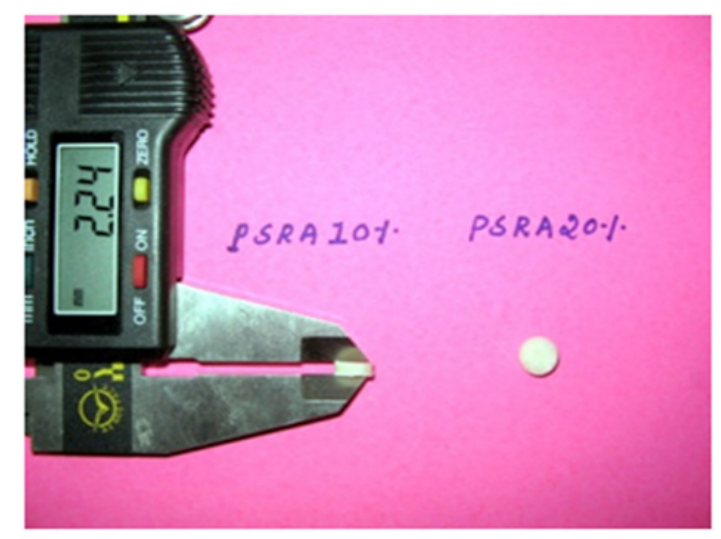

Figure 1: Illustration of the DOX HCL HCL-loaded $10 \% \mathrm{w} / \mathrm{w}$ and $20 \%$ w/w PSRA7/3 implants. the acquired drug was analyzed to be $36.94 \% \pm 0.6 \%$. It was determined that the F3 implant degradation mass loss after 20 days was $90.59 \% \pm 1.6 \%$. A similar result was presented in separate studies that illustrated how the erosion grades of the P (FAD-SA) formulations were measured as weight loss that was prompted by the physicochemical equities of the blended compound. The hydrophilic compound accelerated erosion, while the hydrophobic compound minimized the destruction of P (FAD-SA) materials. ${ }^{17}$

\section{Dissolution studies}

Figure 4 presents the results of the dissolution studies of the DOX.HCl-loaded PSRA7/3 w/w implants. Drug release was carried out in PBS ( $\mathrm{pH}$ 7.4) as a dissolution medium at $37^{\circ} \mathrm{C}$. The $10 \% \mathrm{w} / \mathrm{w}$ loaded implant's mean cumulative drug-release rate in the first 2 days was $0.84 \% \pm 0.5 \%$. Similarly, the $20 \% \mathrm{w} / \mathrm{w}$ drug-loaded implant showed a drug-release rate of $4.04 \% \pm 2.4 \%$. As the amount of $\mathrm{DOX} \cdot \mathrm{HCl}$ increased in the implant, the drug-release rate increased correspondingly. From day 4 , the release curve appeared to show an upturn at a comparativelv faster bace until dav 20. with an average,

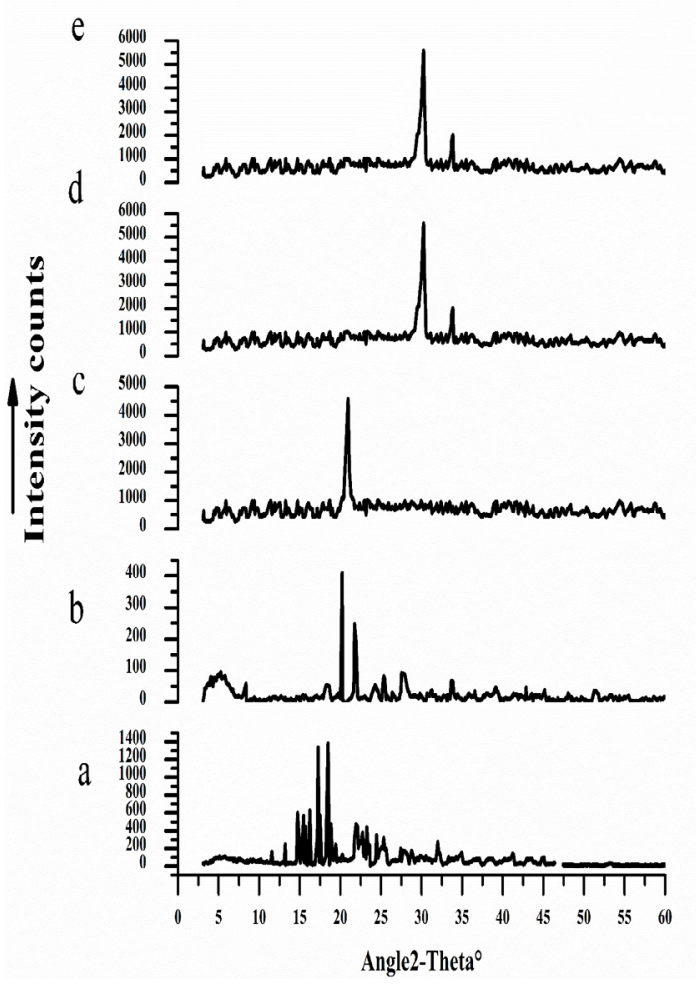

Figure 2: The X-ray diffraction patterns of DOX HCL (a), PSRA7/3 (b), $\beta$-cyclodextrin (c) and drug-loaded implants F2 (d) and $F(e)$. The equipment was operated using a step-scan technique of $0.02(2 \theta)$ increments to measure the crystalline character of the patterns. With respect to the formulations, the implants were powdered well and the crystalline quality of the samples was tested. 


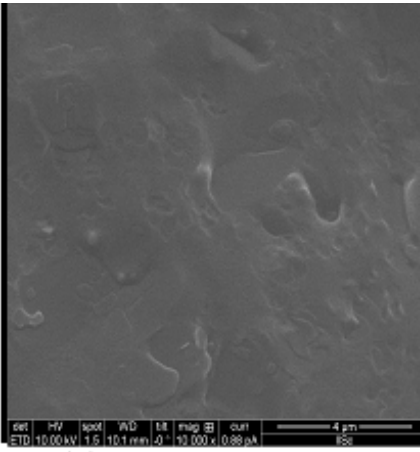

a-Intial

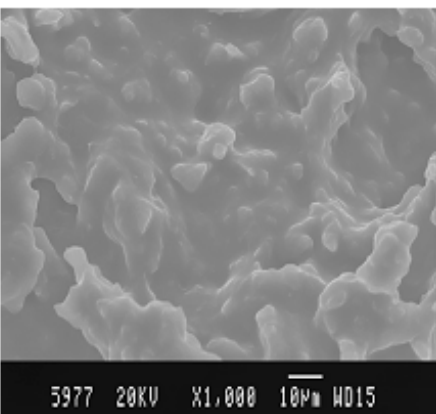

b-after 5 days

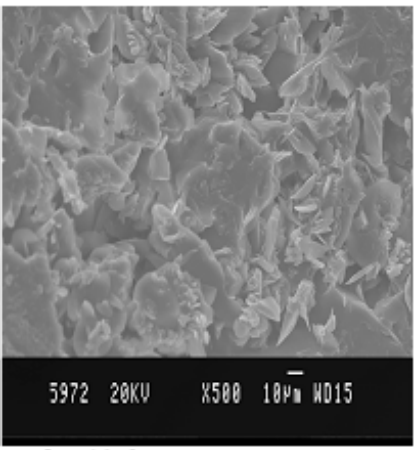

c-after 10 days

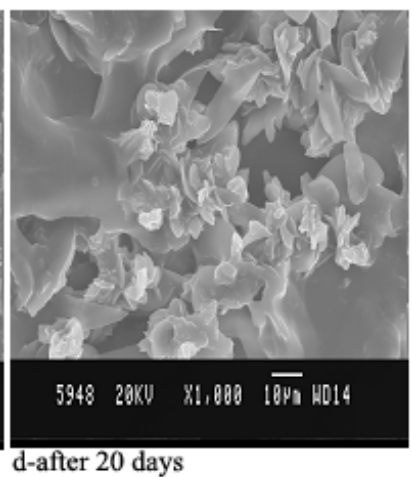

d-after 20 days

Figure 3: SEM photographs of the in vitro hydrolytic cleavage samples of the $20 \% \mathrm{w} / \mathrm{w}$ drug-loaded sample at the initial time point (a) before being exposed to the degradation subject, after day 5 (b), after 10 day (c) and at day 20 (d). The patterns showed the progression of hollow water cavities or large, uneven thicknesses of pores, which might be influenced by the presence of $\beta$-cyclodextrin. This might solubilize the drug during the cleavage of PSRA 7/3 w/w surface erosion; the hydrolytic degradation was investigated. The SEM images were obtained at suitable magnification.

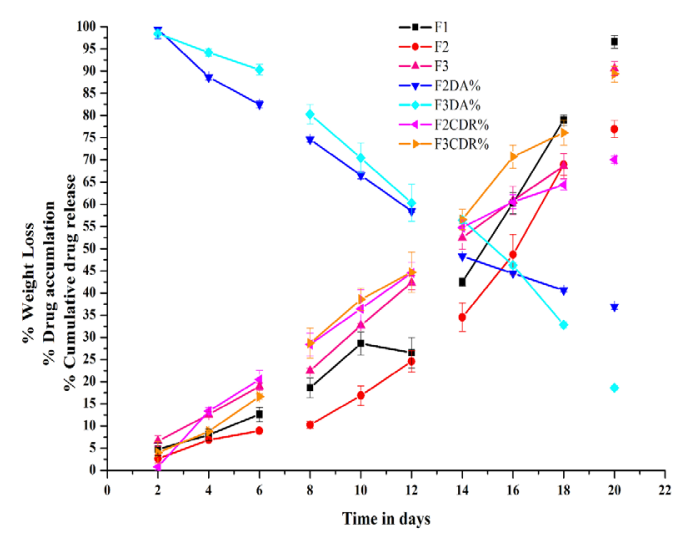

Figure 4: Hydrolytic degradation of the polymer; percent weight loss for F1 (•), F2 (•) and F3 ( $\Delta)$, as well as the percent of drug accumulated during weight loss for F2 $(\nabla)$ and F3

$(\bullet)$. Also presented is the cumulative percent of drug release versus time forF2 $(\triangleleft)$ and F3 $(\triangleright)$. The implants were placed in $20 \mathrm{~mL}$ of phosphate buffered saline (PBS; pH 7.4) in $50 \mathrm{~mL}$ screw-capped bottles. These vials were kept in a horizontal shaker and continuously vibrated at an acceleration of $\mathbf{5 0}$ rpm to activate the hydrolytic degradation, surface erosion, or bulk erosion over 20 days.

sustained drug release of $70.06 \% \pm 1.9 \%$ (F2 10\% w/w) and $89.36 \% \pm 1.8 \%$.

Table 1 shows the macroscopic properties of the blank and drug-loaded implants. Drug-loaded PSRA7/3 with yellow $\beta$-cyclodextrin cylinders, created using a melting procedure, featured a set amount of polymers that varied in the amount of drug that was loaded; they produced $10 \% \mathrm{w} / \mathrm{w}$ and $20 \% \mathrm{w} / \mathrm{w}$. The change in the weight and thickness of the implants was recognized when correlated to the initial amount of drug present; polymers were taken to prepare the formulations. This attribute because of the chain reorientation of the polymeric molecules during heating/melt process, direct freezing and insertion of a plunger to make a

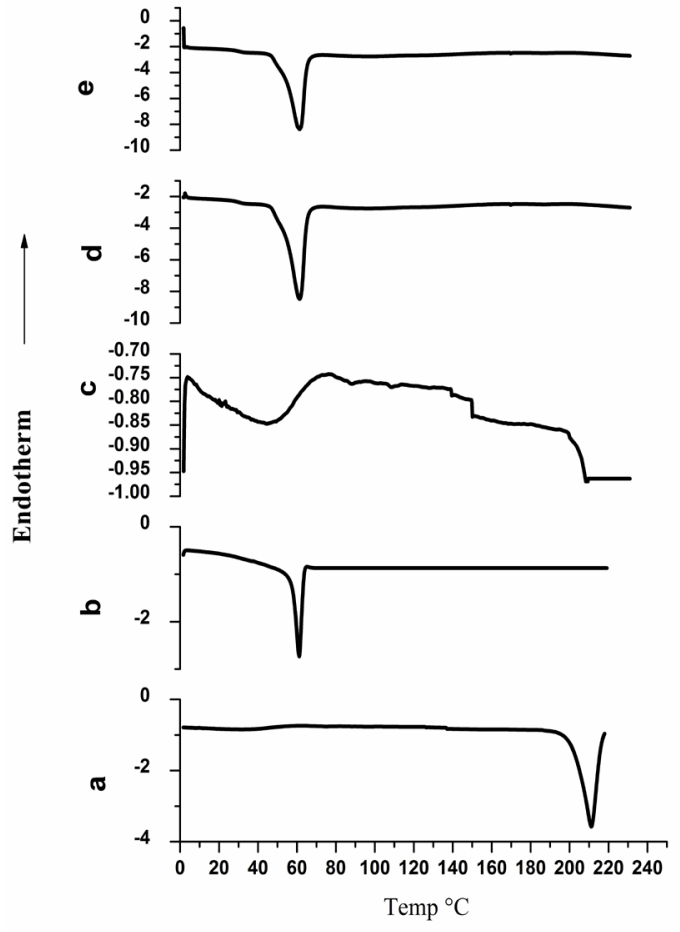

Figure 5: The X-ray diffraction patterns of DOX HCL (a), PSRA7/3 (b), $\beta$-cyclodextrin (c) and drug-loaded implants F2 (d) and $F(e)$. The equipment was operated using a step-scan technique of $0.02(2 \theta)$ increments to measure the crystalline character of the patterns. With respect to the formulations, the implants were powdered well and the crystalline quality of the samples was tested.

compact uniform shape accompanied by solidification of implants. Rapid cooling could influence the implants' solid or rigid structure. Various dimensions of the implant, such as its area, volume and surface area played a significant role in the hydrolytic degradation of the polymer, ultimately influencing the drug's ability to discharge from the cylinder. The differences in the thickness and weight values of the implants are 
displayed in Table 1. $\beta$-cyclodextrin was incorporated in the manufacturing process to enhance the drug's solubility within a polymeric surface and to generate pores that could easily influence water penetration into the implants, while activating the polymer to induce the surface or bulk hydrolytic erosion of the polymer. The DSC analysis (Figure 5) shows that the drug peak disappeared in the process of implants F2 and F3exhibitingthe drug existence in the presence of dissolution, semi crystalline, or drug adsorbed at the amorphous polymer surface. These results were again confirmed with XRD studies (Figure 3), which indicated that the crystalline scattered angle peaks disappeared in the drug-loaded implants. Hydrolytic degradation is the transformation of polymer chain cleavage. They hydrolyze into dicarboxylicacids and hydroxy acid monomers when placed in an aqueous vehicle. Polyanhydride belongs to a class of waterinsoluble hydrophobic polymers. It has a hydrophobic backbone with a hydrolytically labile anhydride and/or ester links. Erosion is the method of transfer that can lead to the loss of polymer mass or monomers. ${ }^{25,26}$ The blank implants showed a faster hydrolytic degradation rate when compared with the drug-loaded implants. The erosion rates of the cylinders were determined to be physicochemical properties of the blended compound, which prompted the observed weight loss. Figure 4 presents the results of the dissolution studies of the DOX $\cdot \mathrm{HCl}$-loaded PSRA7/3 w/w implants. Drug release was carried out in PBS ( $\mathrm{pH}$ 7.4) as the dissolution medium at $37^{\circ} \mathrm{C}$. As Since the percentage of the drug is higher in the polymer, the amount of drug released is faster due to the influence of $\beta$-cyclodextrin polymerinduced drug solubility ${ }^{27}$ and the creation of more water channels. Further, high water uptake produced faster hydrolytic polymer degradation which, in turn, controlled the drug released from cylinder. ${ }^{28}$ This means that $\mathrm{DOX} \cdot \mathrm{HCl}$ is easily soluble in water and normal saline. Degradation transforms the surface (homogenous) and bulk heterogeneous eroding polymers. The time at which the degradation of the full polymer quantity is for high water penetration than the amount of polymer cleavage. It is a continual disposing control cleavage of oligomers into monomers throughout the polymer matrix. Surface cleavage (breakdown) is the hydrolysis of the polymer to the outer surface and the interior matrix remains an under process of cleavage of oligomers followed by monomers. It selects the particular surface erosion process the polymer cleavage should be quicker than water uptake or diffusibility or dissolution. The hydrophobic polyanhydride polymers show how surface disintegration occurs only when the highest water penetration should be at the appearing surface than at the interior of the bulk. ${ }^{29}$ We observed the SEM results of the in vitro release types of the drugloaded 20\% w/w typical F3 formulation at periodic time intervals (a). Figure 4 presents the surface morphology of the intact implant before the in vitro hydrolytic degradation occurred and was characterized as a stable, continuous, smooth and uniform surface (a). The surface

\begin{tabular}{|c|c|c|c|c|c|c|}
\hline Batch & $\begin{array}{l}\text { Polymer } \\
\text { (mg) }\end{array}$ & $\begin{array}{l}\text { DOX.HCL } \\
\text { (mg) }\end{array}$ & $\begin{array}{c}\beta \text {-cyclodextrin } \\
\text { (mg) }\end{array}$ & $\begin{array}{c}\text { Drug loading } \\
\%\end{array}$ & $\begin{array}{c}\text { Implant } \\
\text { weight (mg) }\end{array}$ & $\begin{array}{c}\text { Drug content } \\
\%\end{array}$ \\
\hline $\mathrm{F} 1$ & 180 & - & 20 & - & $120.46 \pm 1.98$ & - \\
\hline F3 & 180 & 20 & 20 & 10 & $142.86 \pm 1.24$ & $98.96 \pm 0.4$ \\
\hline $\mathrm{F} 4$ & 180 & 40 & 20 & 20 & $160.84 \pm 2.46$ & $99.84 \pm 0.8$ \\
\hline Batches & Appearance & Color & Area $\left(\mathrm{cm}^{2}\right)$ & $\begin{array}{c}\text { Thickness }(\mathrm{h}) \\
(\mathrm{cm})\end{array}$ & Volume $\left(\mathrm{cm}^{2}\right)$ & $\begin{array}{c}\text { Surface area } \\
\mathrm{cm}^{2}\end{array}$ \\
\hline F1 & Cylinder & Yellow & $0.346 \pm 0.4$ & $2.06 \pm 0.01$ & $6.27 \pm 0.02$ & $7.01 \pm 0.05$ \\
\hline $\mathrm{F} 2$ & Cylinder & Yellow & $0.468 \pm 0.2$ & $2.24 \pm 0.02$ & $6.48 \pm 0.04$ & $7.06 \pm 0.02$ \\
\hline F3 & Cylinder & Yellow & $0.586 \pm 0.1$ & $3.06 \pm 0.01$ & $9.51 \pm 0.8$ & $7.22 \pm 0.03$ \\
\hline Batches & $\begin{array}{l}\text { Zero order } \\
Q_{t=} Q_{0}+K_{0} t\end{array}$ & $\begin{array}{c}\text { First order } \\
\text { In } Q_{\mathrm{t}=} \ln Q_{\mathrm{o}}+ \\
\mathrm{K}_{\mathrm{o}} \mathrm{t}\end{array}$ & $\begin{array}{c}\text { Higuchi } \\
\mathrm{Q}_{\mathrm{t}=} \mathrm{K}_{\mathrm{H}} \text { sq.tR } \mathrm{tR}^{2}\end{array}$ & $\begin{array}{c}\text { Korsmeyer- } \\
\text { Peppas } \\
Q_{t} / Q_{\text {inf }} K_{k} t^{n} R^{2}\end{array}$ & $\begin{array}{c}\text { Korsmeyer- } \\
\text { Peppas } \\
Q_{\mathrm{t}} / \mathrm{Q}_{\mathrm{inf}=} \mathrm{K}_{\mathrm{k}} \mathrm{t}^{\mathrm{n}} \mathrm{R}^{2} \\
\mathrm{n}=\text { values }\end{array}$ & $\begin{array}{c}P \\
(P \text {-value })\end{array}$ \\
\hline $\mathrm{F} 2$ & 0.934 & 0.845 & 0.863 & 0.9444 & $(n=0.69)$ & $<0.001$ \\
\hline F3 & 0.912 & 0.824 & 0.856 & 0.9064 & $(n=0.64)$ & $<0.003$ \\
\hline
\end{tabular}

The following mathematical equations were used to determine the physical parameters of the implants: $\left(\mathrm{cm}^{2}\right) \mathrm{A}=2 \pi \mathrm{rh}+2 \pi r^{2}$ Volume $\left(\mathrm{cm}^{2}\right)$ $=\pi X r^{2} X h$, Surface area $\left(\mathrm{cm}^{2}\right)=\left[\left(\pi X r^{2} X 2\right)+(2 X \pi X r X h)\right]$. 
morphology of the sample after day 5 slightly influenced the bulk erosion (b). After day 10 and at the conclusion of day 20, the surface morphology showed greater bulk erosion and surface erosion. Highly porous, extend in some hallow carries with an effect of $\beta$-cyclodextrin with a drug in aqueous solution, the compounds readily dissociate and free drug molecules persist in equilibrium with the molecules confined within the $\beta$-cyclodextrin hallow carries may start to PSRA7/3 polymer surface eroding, with further loss of material from the exterior surface and samples diminished further in their size with controlled drug deliver for a prolonged stage of polymer degradation was explained. The same approach used in separate studies indicated that drug delivery is independent of its physicochemical properties and is effectively commanded by erosion. The breakdown of the polymer matrices depends on transfers such as the rate of polymer cleavage, the acceleration of water uptake (at which point the polymer swells), the resulting pore structure and the momentum of oligomer and monomer dispersion from the matrices. Such erosion maintains a continuous surface field and leads to zeroorder drug release. ${ }^{26}$ Thus, the variation in matrix degradation observed between particular combinations of polymer and drug amounts could be in response to the polyanhydride degradation that occurs by surface dissolution and other physicochemical factors that involve transformation and shape degradation. For criterion, the monomers and their structure determine the significant associates. ${ }^{30,31}$

It is actually important to identify the visible and atomic degradation properties of polyanhydrides at a molecular level. The geometry of cylindrical, rod and rectangular dimensions influences the cleavage of polymers (erosion). As the devices increase in thickness, the polymer is slower to cleave to smaller surface areas and accommodates finite water penetration. This is involved in the delayed, controlled drug release that occurs by surface erosion. ${ }^{31}$ There is a chance that this process varies based on hydrophilic high drug loading, the drug's solubility, the molecular weight of the drug and the polymer's solubility, which can intensify the co-adjuvant polymer or the excipients incorporated in the matrices or devices. The drug-release kinetics were established based on in vitro release data by plotting the percent cumulative drug release in association with time; this indicated the gradual and continuous drug releasepatterns that were observed and which were independent of concentration. The Korsmeyer Peppas design showed that drug release occurs through diffusion and erosion process. A value between 0.64-0.69 indicates irregular and relaxation kinetics processes.

\section{CONCLUSION}

Doxorubicin-loaded biodegradable polymeric implants using PSRA $7 / 3$ and other $\beta$-cyclodextrin polymers were blended to enhance drug diffusion. The melting method successfully produced implants. Changes in the dimensions of the implants were observed when correlated to the initial amount of drug and polymers used for formulation development. The XRD and DSC studies of cylinders F2 and F3showed the presence of the drug, the dissolution behavior, the semicrystalline structure and the drug adsorbed at the amorphous polymer surface. The in vitro drug-release studies explained that as the amount of $\mathrm{DOX} \cdot \mathrm{HCl}$ increased in the implant, the drug-release rate increased correspondingly. However, $\beta$-cyclodextrin promoted the release of the drug from the cylindrical device since the percentage of the drug was higher in the polymer; as such, the amount of drug released is higher because the influence of $\beta$-cyclodextrin-induced drug solubility was revealed. The in vitro drug release subjected samples morphology using SEM studies proved that surface morphology of the intact implant before succeeding in vitro hydrolytic degradation characterized as a stable continuous smooth uniform surface. The surface morphology of the sample after day 5 influenced bulk erosion. After day 10 and at the end of day 20 , the surface morphology showed greater bulk and surface erosion. The structure was porous and presented some water cavities'-cyclodextrin was carried out. The drug-release kinetics investigations demonstrated that this occurred independent of concentration and the Korsmeyer Peppas design provided a good prepared for the drug release implants with greater regression coefficients in corresponding to alternative designs. These prepared implants may function as local drug-delivery systems using polyanhydride polymers as a safe substitute to transport anticancer agents to tumors. Investigations with in vitro cell lines and animal subjects will be carried out in future studies.

\section{ACKNOWLEDGEMENT}

The authors acknowledge the Deanship of Scientific Research at King Faisal University for providing financial support under the Nasher Track (grant number: 186248).

\section{CONFLICT OF INTEREST}

The authors declare that there are no conflicts of interest.

This article does not contain any studies with human or animal subjects performed by any of the authors. 


\section{ABBREVIATIONS}

(DOX·HC1): Doxorubicin; (FTIR) spectroscopy: Fourier Transform Infrared; (XRD): X-ray diffraction; DSC: Differential scanning calorimetry; SEM: Scanning Electronic Microscopy; SA: Synthesizedsebacic acid; RA: Ricinoleic acid; PCPP-SA: poly ([carboxy phenoxy] propane-sebacic acid); HPA: Hydroxy stearic acid.

\section{REFERENCES}

1. Basu A, Domb AJ. Recent Advances in Polyanhydride Based Biomaterials. Advanced Materials. 2018;30(41):1706815.

2. Doppalapudi S, Jain A, Domb AJ, Khan W. Biodegradable polymers for targeted delivery of anti-cancer drugs. Expert Opinion on Drug Delivery. 2016;13(6):891-909.

3. Doppalapudi S, Jain A, Khan W, Domb AJ. Biodegradable polymers-an overview. Polymers for Advanced Technologies. 2014;25(5):427-35.

4. Rosen HB, Chang J, Wnek G, Linhardt R, Langer R. Bioerodible polyanhydrides for controlled drug delivery. Biomaterials. 1983;4(2):131-3.

5. Brenza TM, Schlichtmann BW, Bhargavan B, Vela RJE, Nelson RD, Panthani $M G$, et al. Biodegradable polyanhydride-based nanomedicines for blood to brain drug delivery. Journal of Biomedical Materials Research Part A. 2018;106(11):2881-90.

6. Domb AJ, Nudelman R. Biodegradable polymers derived from natural fatty acids. Journal of Polymer Science Part A: Polymer Chemistry. 1995;33(4):717-25.

7. Leong K, D'Amore P, Marletta M, Langer R. Bioerodible polyanhydrides as drug-carrier matrices. II. Biocompatibility and chemical reactivity. Journal of Biomedical Materials Research. 1986;20(1):51-64.

8. Domb A, Maniar M, Bogdansky S, Chasin M. Drug delivery to the brain using polymers. Critical Reviews in Therapeutic Drug Carrier Systems. 1991;8(1):117.

9. Brem H, Piantadosi S, Burger P, Walker M, Selker R, Vick N, et al. Placebocontrolled trial of safety and efficacy of intraoperative controlled delivery by biodegradable polymers of chemotherapy for recurrent gliomas. The Lancet. 1995;345(8956):1008-12.

10. Li LC, Deng J, Stephens D. Polyanhydride implant for antibiotic delivery-from the bench to the clinic. Advanced Drug Delivery Reviews. 2002;54(7):963-86.

11. Göpferich A. Bioerodible implants with programmable drug release. Journal of Controlled Release. 1997;44(2-3):271-81.

12. Vogelhuber W, Spruß T, Bernhardt G, Buschauer A, Göpferich A. Efficacy of BCNU and paclitaxel loaded subcutaneous implants in the interstitial chemotherapy of U-87 MG human glioblastoma xenografts. International Journal of Pharmaceutics. 2002;238(1):111-21

13. Shikanov A, Vaisman B, Shikanov S, Domb AJ. Efficacy of poly (sebacic acid-co-ricinoleic acid) biodegradable delivery system for intratumoral delivery of paclitaxel. Journal of Biomedical Materials Research Part A: An Official Journal of the Society for Biomaterials, The Japanese Society for
Biomaterials and The Australian Society for Biomaterials and the Korean Society for Biomaterials. 2010;92(4):1283-91.

14. Jaszcz K. Effect of basic factors of preparation on characteristics, hydrolytic degradation and drug release from poly (ester-anhydride) microspheres. International Journal of Polymeric Materials and Polymeric Biomaterials. 2014;63(2):97-106.

15. Krasko MY, Shikanov A, Ezra A, Domb AJ. Poly (ester anhydride) s prepared by the insertion of ricinoleic acid into poly (sebacic acid). Journal of Polymer Science Part A: Polymer Chemistry. 2003;41(8):1059-69.

16. Sokolsky-Papkov M, Agashi K, Olaye A, Shakesheff K, Domb AJ. Polymer carriers for drug delivery in tissue engineering. Advanced Drug Delivery Reviews. 2007;59(4-5):187-206.

17. Park ES, Maniar M, Shah JC. Influence of physicochemical properties of model compounds on their release from biodegradable polyanhydride devices. Journal of Controlled Release. 1997;48(1):67-78.

18. Uhrich KE, Cannizzaro SM, Langer RS, Shakesheff KM. Polymeric systems for controlled drug release. Chemical Reviews. 1999;99(11):3181-98.

19. Domb A, Langer R. Polyanhydrides. I. Preparation of high molecular weight polyanhydrides. Journal of Polymer Science Part A: Polymer Chemistry. 1987;25(12):3373-86.

20. Domb AJ, Gallardo CF, Langer R. Poly (anhydrides). 3. Poly (anhydrides) based on aliphatic-aromatic diacids. Macromolecules. 1989;22(8):3200-4.

21. Patil R, Portilla-Arias J, Ding H, Konda B, Rekechenetskiy A, Inoue S, et al. Cellular delivery of doxorubicin via $\mathrm{pH}$-controlled hydrazone linkage using multifunctional nano vehicle based on poly ( $\beta$-L-malic acid). International Journal of Molecular Sciences. 2012;13(9):11681-93.

22. Shuai X, Ai H, Nasongkla N, Kim S, Gao J. Micellar carriers based on block copolymers of poly ( $\varepsilon$-caprolactone) and poly (ethylene glycol) for doxorubicin delivery. Journal of Controlled Release. 2004;98(3):415-26.

23. Dhanikula $A B$, Panchagnula R. Localized paclitaxel delivery. International Journal of Pharmaceutics. 1999;183(2):85-100.

24. Hiremath J, Kusum DV, Devi K, Domb A. Biodegradable poly (sebacic acid-coricinoleic-ester anhydride) tamoxifen citrate implants: Preparation and in vitro characterization. Journal of Applied Polymer Science. 2008;107(5):2745-54.

25. Jain JP, Modi S, Domb A, Kumar N. Role of polyanhydrides as localized drug carriers. Journal of Controlled Release. 2005;103(3):541-63.

26. Tamada J, Langer R. Erosion kinetics of hydrolytically degradable polymers. Proceedings of the National Academy of Sciences. 1993;90(2):552-6.

27. Brewster ME, Loftsson T, Estes KS, Lin JL, Fridriksdóttir H, Bodor N. Effect of various cyclodextrins on solution stability and dissolution rate of doxorubicin hydrochloride. International Journal of Pharmaceutics. 1992;79(1-3):289-99.

28. Tiwari G, Tiwari R, Rai AK. Cyclodextrins in delivery systems: Applications. Journal of Pharmacy and Bioallied Sciences. 2010;2(2):72.

29. Göpferich A, Teßmar J. Polyanhydride degradation and erosion. Advanced Drug Delivery Reviews. 2002;54(7):911-31.

30. Mathiowitz E, Ron E, Mathiowitz G, Amato C, Langer R. Morphological characterization of bioerodible polymers. 1 . Crystallinity of polyanhydride copolymers. Macromolecules. 1990;23(13):3212-8.

31. Akbari H, D'Emanuele A, Attwood D. Effect of geometry on the erosion characteristics of polyanhydride matrices. International Journal of Pharmaceutics. 1998;160(1):83-9.

\section{SUMMARY}

Doxorubicin-loaded biodegradable polymeric implants using PSRA 7/3 and other $\beta$-cyclodextrin polymers were blended to enhance drug diffusion. The melting method successfully produced implants. Changes in the dimensions of the implants were observed when correlated to the initial amount of drug and polymers used for formulation development. In vitro drug release studies illustrated that the drug-release rate was faster in association with increasing amounts of doxorubicin in the implant. 
PICTORIAL ABSTRACT

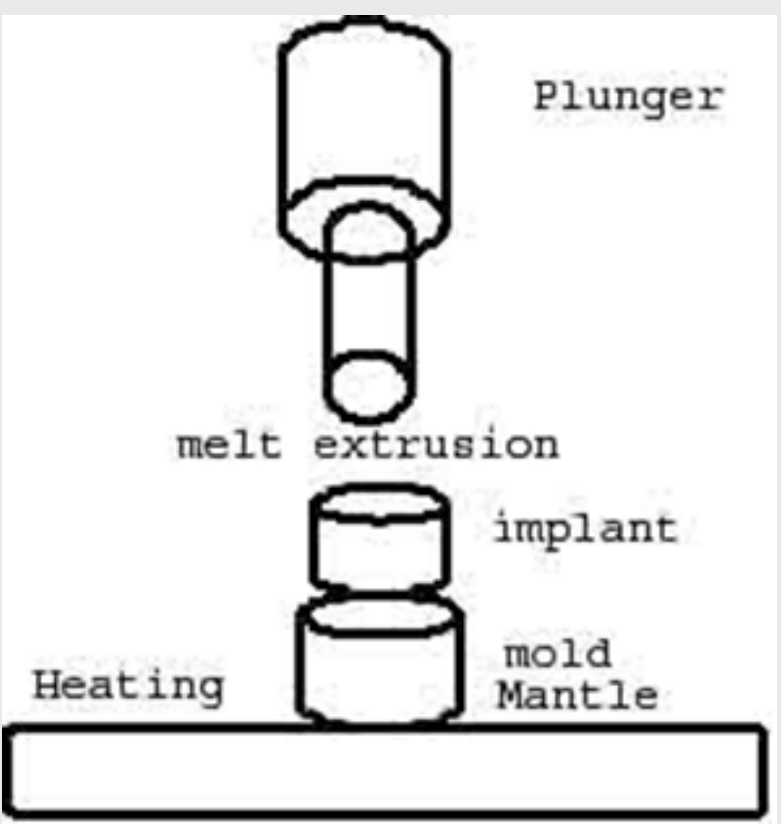

Dr. Sree Harsha received his (ranked top 5) Master of Pharmacy Degree and subsequently earned a doctorate in Pharmaceutics from Rajiv Gandhi University of Health Sciences, Bangalore, India in 2006. He came to King Faisal University in 2007 as an assistant professor in the Department of Pharmaceutical Sciences, bringing with him several years' worth of teaching experience in fundamentals of pharmaceutics and drug delivery systems. He was actively participated in Accreditation Council of Pharmacy Education (ACPE) and Canadian Council for the Accreditation of Pharmacy Programs (CCAPP). His primary area of focus is pharmaceutical technology and novel/ targeted drug delivery systems. For this research, he received grants (30 number) from Deanship of Scientific research, King Faisal University. The author contributed so far to 80 peer-reviewed full papers on a variety of topics in lung targeting, topical drug delivery and mucoadhesive drug delivery systems, He has contributed in writing a book chapter titled "Targeted Drug Delivery System" and "Microspheres" in Textbook of Industrial Pharmacy, Publisher-Orient Longman Private Ltd. In addition, he is an Ad-hoc reviewer for scientific journals. He has attended many seminars and Workshop both national and international on Pharmaceutical Technology and Public health issues.

Cite this article: Sreeharsha N, Hiremath JG, Al-Dhubiab BE, Meravanige G, Karnati RK, Attimarad M, Nair AB, Venugopal KN, Morsy MA, Pottathil S, Khan S, Molugulu N. Fabrication of Poly (sebacic acid-co-ricinoleic-ester anhydride) with $\beta$-cyclodextrin-loaded doxorubicin Implants and in vitro characterization. Indian $\mathrm{J}$ of Pharmaceutical Education and Research. 2020;54(3):826-34. 